\title{
A CONJECTURE ON POINCARÉ-BETTI SERIES OF MODULES OF DIFFERENTIAL OPERATORS ON A GENERIC HYPERPLANE ARRANGEMENT
}

\author{
JAN SNELLMAN
}

\begin{abstract}
Holm [7 6] studied modules of higher order differential operators (generalizing derivations) on generic (central) hyperplane arrangements. We use his results to determine the Hilbert series of these modules. We also give a conjecture about the Poincaré-Betti series; these are known for the module of derivations through the work of Yuzvinsky 13 and Rose and Terao 11.
\end{abstract}

\section{INTRODUCTION}

The module of derivations $\mathcal{D}^{(1)}(\mathcal{A})$ of a hyperplane arrangement $\mathcal{A} \in \mathbb{C}^{n}$ (henceforth called an $n$-arrangement) is an interesting and much studied object [10, 9, 8. In particular, the question whether this module is free, for various classes of arrangements, has received great attention.

On the other hand, the module of higher differential operators $\mathcal{D}^{(m)}(\mathcal{A})$ received their first incisive treatment in the PhD thesis of Pär Holm [6]. The deepest result in that work concerns so-called generic arrangements, which are arrangements where every intersection of $s \leq n$ hyperplanes in $\mathcal{A}$ have the expected codimension $s$. Holm gave a concrete generating set for $\mathcal{D}^{(m)}(\mathcal{A})$, proved an extension of Saito's determinental criteria for freeness of derivations, and used these results to tackle the question of higher order freeness for generic arrangements, i.e. the question when $\mathcal{D}^{(m)}(\mathcal{A})$ is a free module (in which case we say that $\mathcal{A}$ is $m$-free). In brief, he showed that

(i) all 2-arrangements are $m$-free for all $m$,

(ii) all $n$-arrangements with $|A| \leq n$ are $m$-free for all $m$,

(iii) if $n \geq 3$ and $r>n$ and $m<r-n+1$, then $\mathcal{A}$ is not $m$-free,

(iv) if $n \geq 3$ and $r>n$ and $m=r-n+1$, then $\mathcal{A}$ is $m$-free.

He conjectured that if $n \geq 3$ and $r>n$ and $m>r-n+1$ then $\mathcal{A}$ is $m$-free.

For $m=1$, (iii) becomes $r>n \geq 3$. Yuzvinsky [13] and independently Terao and Rose [1] have showed that the modules of derivations of generic arrangements are non-free, with a minimal free resolution of length $n-2$. More precisely, they have showed that the graded Betti numbers are given

Date: November 15, 2018.

1991 Mathematics Subject Classification. 13N10, 14J70.

Key words and phrases. Ring of differential operators, hyperplane arrangement, free resolution. 
by

$$
\beta_{k, u}= \begin{cases}1 & \text { if } k=0 \text { and } u=1 \\
\left(\begin{array}{c}
r \\
n-k
\end{array}\right)\left(\begin{array}{c}
r-n+k-2 \\
k-1
\end{array}\right) & \text { if } u+n-r-1=0 \\
0 & \text { otherwise }\end{cases}
$$

so that the Poincaré-Betti series can be expressed as

$$
b+\left[t^{n-r-1}(1+b t)^{r-1}\right] \odot(1-t)^{n-r},
$$

where $t$ enumerates homological degree, $b$ ring degree, and $\odot$ denotes Hadamard product of power series.

This articles contains a short exposé of various ways of calculating modules of differential operators (on a computer), a brief review of the work of Holm, and finally some conjectures supported by extensive computer experiments. The most important one is the conjectured formula for the Poincaré-Betti series of $\mathcal{D}^{(m)}(\mathcal{A})$ when $\mathcal{A}$ is a generic $n$-arrangement with $|\mathcal{A}|=r, 3 \leq n, r \geq m+n$ :

$$
\begin{aligned}
\mathcal{P}\left(\mathcal{D}\left(\mathcal{A}_{n, r}\right)^{(m)}\right)=b^{m}+ & \\
& +\left\{t^{-r+n-1}\left((1+b t)^{m}-(b t)^{m}\right)(1+b t)^{r-m}\right\} \odot(1-t)^{m-r+n-1} .
\end{aligned}
$$

\section{Notation}

For basic terminology regarding hyperplane arrangements, we refer to Orlik and Terao's treatise [10. For more details on the Grothendieck ring of differential operators, see for instance the PhD thesis by Holm [6], or [2, 4].

Let $\mathcal{A}$ be an affine central hyperplane arrangement in $\mathbb{C}^{n}$, with defining polynomial $p=\prod_{i=1}^{r} p_{i} \in S=\mathbb{C}\left[x_{1}, \ldots, x_{n}\right]$. We let $\mathcal{D}(S)$ denote the Weyl algebra of differential operators on $S$. This is the set of all finite $S$-linear combinations

$$
\delta=\sum_{\alpha \in \mathbb{N}^{n}} c_{\alpha} \partial^{\alpha}
$$

An element of $\mathcal{D}(S)$ can be regarded as a partial differential operator with polynomial coefficients, and thus it induces an $S$-algebra endomorphism. We use the notation $Q * v$ to denote the action of $Q \in \mathcal{D}(S)$ on $v \in S$.

$\mathcal{D}(S)$ is an $S$-module in a natural way: the action is given by

$$
q \sum_{\alpha \in \mathbb{N}^{n}} c_{\alpha} \partial^{\alpha}=\sum_{\alpha \in \mathbb{N}^{n}} q c_{\alpha} \partial^{\alpha}
$$

If in (11) all $\alpha$ with $c_{\alpha} \neq 0$ have total degree $m$ we say that $\delta$ is a homogeneous $m$ 'th order operator, and write $|\delta|=m$, or $\delta \in \mathcal{D}^{(m)}(S)$. If, in addition, all $c_{\alpha}$ occurring in (11) are homogeneous polynomials of total degree $v$, we say that $\delta$ is homogeneous of polynomial degree $v$. Thus $\mathcal{D}(S)$ is a bi-graded $S$ module, where we use the convention that $x^{\alpha} \partial^{\beta}$ has bi-grade $(k, m)=(|\alpha|,|\beta|)$. 
Let $m$ be a positive integer. We put

$$
\begin{array}{r}
\mathcal{D}(\mathcal{A})=\left\{\delta=\sum_{\alpha \in \mathbb{N}^{n}} c_{\alpha} \partial^{\alpha} \mid c_{\alpha} \in S, \delta *\langle p\rangle \subseteq\langle p\rangle\right\} \\
\mathcal{D}^{(m)}(\mathcal{A})=\left\{\delta=\sum_{|\alpha|=m} c_{\alpha} \partial^{\alpha} \mid c_{\alpha} \in S, \delta *\langle p\rangle \subseteq\langle p\rangle\right\}
\end{array}
$$

In particular, $\mathcal{D}^{(1)}(\mathcal{A})$ is the much-studied module of derivations of $\mathcal{A}$.

It is a fact that $\mathcal{D}^{(m)}(\mathcal{A})$ is a graded $S$-module, where the $\mathbb{N}$-grading is given by polynomial degree. Holm [7] showed that $\mathcal{D}(\mathcal{A})=\bigoplus_{m \geq 0} \mathcal{D}^{(m)}(\mathcal{A})$. Hence, the $S$-module $\mathcal{D}(\mathcal{A})$ is bi-graded, and hence so is all Tor modules. The $S$-module $\mathcal{D}(\mathcal{A})$ is not necessarily finitely generated, but every $\mathcal{D}^{(m)}(\mathcal{A})$ is. Consequently we can calculate the graded minimal free resolution

$$
0 \leftarrow \mathcal{D}^{(m)}(\mathcal{A}) \leftarrow \bigoplus_{i} \beta_{1, i}^{(m)} S(-i) \leftarrow \cdots \leftarrow \bigoplus_{i} \beta_{\ell, i}^{(m)} S(-i) \leftarrow 0
$$

where, by the Hilbert syzygy theorem, $\ell \leq n$. We define the Poincaré-Betti series and Hilbert series of $\mathcal{D}^{(m)}(\mathcal{A})$ and of $\mathcal{D}(\mathcal{A})$ by

$$
\begin{aligned}
\mathcal{P}\left(\mathcal{D}^{(m)}(\mathcal{A})\right)(b, t) & =\sum_{j, i} \beta_{j, i}^{(m)} b^{i} t^{j} \\
\mathcal{P}(\mathcal{D}(\mathcal{A}))(a, b, t) & =\sum_{m} a^{m} \mathcal{P}\left(\mathcal{D}^{(m)}(\mathcal{A})\right)(b, t) \\
\mathcal{H}(\mathcal{D}(\mathcal{A}))(a, b) & =(1-b)^{-n} \mathcal{P}(\mathcal{D}(\mathcal{A}))(a, b,-1) \\
\mathcal{H}\left(\mathcal{D}^{(m)}(\mathcal{A})\right)(b) & =(1-b)^{-n} \mathcal{P}\left(\mathcal{D}^{(m)}(\mathcal{A})\right)(b,-1)
\end{aligned}
$$

2.1. Additional notation. We define $\left(\left(\begin{array}{l}a \\ b\end{array}\right)\right)=\left(\begin{array}{c}a+b-1 \\ b\end{array}\right)$, i.e. $\left(\left(\begin{array}{l}a \\ b\end{array}\right)\right)$ is the number of multisets of weight $b$ on an $a$-set.

If $f(t)=\sum_{i=0}^{\infty} c_{i} t^{i}$ is a formal power series in $t$, with coefficients in some commutative ring, we put $\left[t^{\ell}\right] f(t)=c_{\ell}$.

Definition 1. Let $R$ be a commutative ring and let $f, g \in R\left[\left[t^{-1}, t\right]\right]$ be two formal Laurent series, i.e.

$$
f=\left(\sum_{i=-\infty}^{\infty} a_{i} t^{i}\right), \quad g=\left(\sum_{i=-\infty}^{\infty} b_{i} t^{i}\right) .
$$

We define the Hadamard product of $f$ and $g$ by

$$
f \odot g=\sum_{i=-\infty}^{\infty} a_{i} b_{i} t^{i} .
$$

\section{Calculating modules of Differential operators on HYPERPLANE ARRANGEMENTS}

We shall review some methods of calculating generators of the $S$-module $\mathcal{D}^{(m)}(\mathcal{A})$. 
3.1. The "Jacobian" method. First, we describe the most straightforward method, a slight variant of which is used in the Macaulay 2 package "D-modules.m2" [12] by Harry Tsai and Anton Leykin.

Lemma 2. Let $\mathcal{D}^{(m)}(S) \ni \delta=\sum_{|\alpha|=m} c_{\alpha} \partial^{\alpha}$ be homogeneous of polynomial degree $v$. Then $\delta \in \mathcal{D}^{(m)}(\mathcal{A})$ iff $\delta *\left(x^{\beta} p\right) \in\langle p\rangle$ for all $|\beta|<v$.

Corollary 3. Let $m$ be a positive integer. Let $G$ be a row matrix whose entries are $\left\{\partial^{\alpha}|| \alpha \mid=m\right\}$, let $H$ be a column matrix whose entries are $\left\{x^{\beta}|| \beta \mid<m\right\}$, and let $A$ be the matrix indexed by $G$ and $H$ where the $\left(\partial^{\alpha}, x^{\beta}\right)$ entry is $\partial^{\alpha} *\left(x^{\beta} p\right)$. Let $B=[A \mid p I]$, where $I$ is the identity matrix of appropriate dimension. Then the syzygy module of the columns of $B$ correspond to $\mathcal{D}^{(m)}(\mathcal{A})$. More precisely, if

$$
[A \mid p I]\left[\begin{array}{c}
\mathbf{u} \\
\mathbf{w}
\end{array}\right]=\mathbf{0}
$$

then

$$
\sum_{\alpha} u_{\alpha} \partial_{\alpha} \in \mathcal{D}^{(m)}(\mathcal{A})
$$

and this is an isomorphism of $S$-modules.

Example 4. Suppose that $S=\mathbb{C}\left[x_{1}, x_{2}\right]$ and that $p=x_{1}$. For $m=2$ the matrix $A$ is

\begin{tabular}{c|ccc} 
& $\partial_{1}^{2}$ & $\partial_{1} \partial_{2}$ & $\partial_{2}^{2}$ \\
\hline 1 & 0 & 0 & 0 \\
$x_{1}$ & 2 & 0 & 0 \\
$x_{2}$ & 0 & 1 & 0
\end{tabular}

so in order to calculate $\mathcal{D}^{(2)}(\mathcal{A})$ we should calculate the syzygies of the columns of

$$
B=\left(\begin{array}{cccccc}
0 & 0 & 0 & x_{1} & 0 & 0 \\
2 & 0 & 0 & 0 & x_{1} & 0 \\
0 & 1 & 0 & 0 & 0 & x_{1}
\end{array}\right)
$$

A generating set is

$$
\left[\begin{array}{l}
0 \\
0 \\
1 \\
0 \\
0 \\
0
\end{array}\right],\left[\begin{array}{c}
-1 / 2 x_{1} \\
0 \\
0 \\
0 \\
1 \\
0
\end{array}\right],\left[\begin{array}{c}
0 \\
-x_{1} \\
0 \\
0 \\
0 \\
1
\end{array}\right],
$$

so $\mathcal{D}^{(2)}(\mathcal{A})$ is generated by $\partial_{2}^{2},-1 / 2 x_{1} \partial_{1}^{2}$, and $-x_{1} \partial_{1} \partial_{2}$.

3.2. The method of intersecting modules. The following result is proved in [7.

Theorem 5. $\mathcal{D}^{(m)}(p)=\cap_{i=1}^{r} \mathcal{D}^{(m)}\left(p_{i}\right)$.

Holm also showed how to calculate $\mathcal{D}^{m}\left(p_{i}\right)$. Let $q \in S_{1}$ be a linear form, and let $H \subset \mathbb{C}^{n}$ be its associated linear variety (a hyperplane through the origin). 
Definition 6. Let $V$ be the $\mathbb{C}$-vector space $V=\sum_{i=1}^{n} \mathbb{C} \partial^{i}$ and define

$$
V_{H}=\left\{\sum_{i=1} b_{i} \partial_{i} \mid\left(b_{1}, \ldots, b_{n}\right) \in H\right\} .
$$

Then $V_{H}$ is a codimension 1 subspace of $V$.

Lemma 7. Let $\mathcal{N}$ be the module of derivations annihilating $q$. Then $\mathcal{N}=$ $S V_{H}$, and if $\delta$ is any derivation such that $\delta * q=a q, a \in \mathbb{C}^{*}$, then $\mathcal{D}^{(1)}(q)=$ $\mathcal{N}+S \delta$.

Proposition 8. Let $M=\left\{\delta_{1}, \ldots, \delta_{n}\right\}$ be a basis for $V$ such that $\left\{\delta_{1}, \ldots, \delta_{n-1}\right\}$ is a basis for $V_{h}$. Then $N=\left\{\delta_{1}, \ldots, \delta_{n-1}, q \delta_{n}\right\}$ generates $\mathcal{D}^{(1)}(q)$, and

$$
\mathcal{D}^{(m)}(q)=\sum_{\substack{|\alpha|=m \\ \alpha_{n}=0}} S \delta^{\alpha}+\sum_{\substack{|\alpha|=m \\ \alpha_{n}>0}} S q \delta^{\alpha}
$$

The above results can be succinctly summarized as follows: let $A(p)$ be the $n \times n$ coordinate matrix of $N$, i.e. the matrix formed by the coordinate vectors of elements of $N$, and let $S^{m} A(p)$ denote the $m$ 'th symmetric power. Let $B^{m}(p)$ be the result of replacing any occurrence of $q^{i}$, with $i>1$, by $q$. In other words, if $A(p)$ is regarded as the matrix of an endomorphism $\phi: S^{n} \rightarrow$ $S^{n}$, then $S^{m} A(p)$ is the matrix of the endomorphism $S^{m} \phi: S^{m} S^{n} \rightarrow S^{m} S^{n}$, and $B^{m}(p)$ is the matrix of the associated endomorphism on $S^{m} T$, where $T=S /\left(q-q^{2}\right)$.

Example 9 (Example 4 cont.). If $S=\mathbb{C}\left[x_{1}, x_{2}\right]$ and $p=x_{1}$, then $V_{H}$ is spanned by $\partial_{2}$. Hence, we can take $M=\left\{\partial_{2}, \partial_{1}\right\}$ and $N=\left\{\partial_{2}, x_{1} \partial_{1}\right\}$, so if we order the monomials of degree two as $x_{1}^{2}, x_{1} x_{2}, x_{2}$ then

$$
A=\left[\begin{array}{cc}
0 & x_{1} \\
1 & 0
\end{array}\right], \quad S^{2} A=\left[\begin{array}{ccc}
0 & 0 & x_{1}^{2} \\
0 & x_{1} & 0 \\
1 & 0 & 0
\end{array}\right], \quad B=\left[\begin{array}{ccc}
0 & 0 & x_{1} \\
0 & x_{1} & 0 \\
1 & 0 & 0
\end{array}\right] \text {. }
$$

Thus we recover the result that $\mathcal{D}^{(2)}\left(x_{1}\right)$ is generated by

$$
\left[\partial^{2}, \partial_{1} \partial_{2}, \partial_{2}^{2}\right] B=\left[\partial_{2}^{2}, x_{1} \partial_{1} \partial_{2}, x_{1} \partial_{1}^{2}\right] .
$$

Now recall (see for instance [1, Theorem 3.8.3]) the following method of computing intersection of submodules of free modules. Suppose that $M_{1}, \ldots, M_{\ell}$ are submodules of the free module $S^{s}$, that $\underline{\mathbf{e}}=\left[\mathbf{e}_{1}, \ldots, \mathbf{e}_{s}\right]$ is a basis of $S^{s}$, and that the matrix $A_{i}$ consists of the coordinate vectors (as column vectors) for a generating set of $M_{i}$. Then the truncations of the syzygies of the matrix

$$
\left[\begin{array}{cccccc}
I_{s} & A_{1} & 0 & 0 & \cdots & 0 \\
I_{s} & 0 & A_{2} & 0 & \cdots & 0 \\
\vdots & \vdots & \vdots & \ddots & \cdots & \vdots \\
I_{s} & 0 & 0 & 0 & \cdots & A_{s}
\end{array}\right]
$$

correspond to elements in $\cap_{i=1}^{s} M_{i}$. 
Example 10. We have that $\mathcal{D}^{(2)}\left(x_{1} x_{2}\right)=\mathcal{D}^{(2)}\left(x_{1}\right) \cap \mathcal{D}^{(2)}\left(x_{2}\right)$. With respect to the basis $\left[\partial_{1}, \partial_{1} \partial_{2}, \partial_{2}^{2}\right]$ for $\mathcal{D}^{(2)}(S)$ the matrices of $\mathcal{D}^{(2)}\left(x_{1}\right)$ and $\mathcal{D}^{(2)}\left(x_{2}\right)$ can be taken to be

$$
\left[\begin{array}{ccc}
x_{1} & 0 & 0 \\
0 & x_{1} & 0 \\
0 & 0 & 1
\end{array}\right] \text { and }\left[\begin{array}{ccc}
1 & 0 & 0 \\
0 & x_{2} & 0 \\
0 & 0 & x_{2}
\end{array}\right]
$$

The syzygies of the matrix

$$
\left[\begin{array}{ccccccccc}
1 & 0 & 0 & x_{1} & 0 & 0 & 0 & 0 & 0 \\
0 & 1 & 0 & 0 & x_{1} & 0 & 0 & 0 & 0 \\
0 & 0 & 1 & 0 & 0 & 1 & 0 & 0 & 0 \\
1 & 0 & 0 & 0 & 0 & 0 & 1 & 0 & 0 \\
0 & 1 & 0 & 0 & 0 & 0 & 0 & x_{2} & 0 \\
0 & 0 & 1 & 0 & 0 & 0 & 0 & 0 & x_{2}
\end{array}\right]
$$

are generated by

$$
\left[\begin{array}{c}
0 \\
0 \\
-x_{2} \\
0 \\
0 \\
x_{2} \\
0 \\
0 \\
1
\end{array}\right],\left[\begin{array}{c}
-x_{1} \\
0 \\
0 \\
1 \\
0 \\
0 \\
x_{1} \\
0 \\
0
\end{array}\right],\left[\begin{array}{c}
0 \\
x_{1} x_{2} \\
0 \\
0 \\
-x_{2} \\
0 \\
0 \\
-x_{1} \\
0
\end{array}\right],
$$

so the intersection of the two modules is generated by

$$
\left[\begin{array}{c}
0 \\
0 \\
-x_{2}
\end{array}\right],\left[\begin{array}{c}
-x_{1} \\
0 \\
0
\end{array}\right],\left[\begin{array}{c}
0 \\
x_{1} x_{2} \\
0
\end{array}\right],
$$

and $\mathcal{D}^{(2)}\left(x_{1} x_{2}\right)$ is generated by $-x_{2} \partial_{2},-x_{1} \partial_{1}, x_{1} x_{2} \partial_{1} \partial_{2}$.

3.3. Calculating modules of differential operators on generic arrangements. Holm [7] gives the following method for constructing a (not necessarily minimal) generating set of $\mathcal{D}^{(m)}(\mathcal{A})$, when $\mathcal{A}$ is a generic $n$ arrangement.

3.3.1. The case $r>n$. Holm showed that for any positive $m$, and for any central arrangement $\mathcal{A}$, the modified Euler derivation

$$
\varepsilon_{m}=\sum_{|\alpha|=m} \frac{m !}{\alpha !} x^{\alpha} \partial^{\alpha}
$$

belongs to $\mathcal{D}^{(m)}(\mathcal{A})$. He then proceeded to find other generators as follows.

Recall the definition of $V$ and of $V_{H}$ from definition [6. We let $H_{i}$ be the hyperplane associated with the linear form $p_{i}$, and put $V_{i}=V_{H_{i}}$. Choose a basis element for each intersection of $n-1$ of the $V_{i}$ 's (by genericity, this intersection is 1-dimensional) and let $M=\left\{\delta_{1}, \ldots, \delta_{t}\right\}$, with $t=\left(\begin{array}{c}r \\ n-1\end{array}\right)$, be the set of all of these. We define a subset $D$ of the derivations on $S$ by

$$
D=\left\{P_{1} \delta_{1}, \ldots, P_{t} \delta_{t}\right\}
$$


where each $P_{i}$ is the product of those $p_{j}$ :s which are not annihilated by $\delta_{i}$. If all $p_{j}$ :s are annihilated by $\delta_{i}$, we put $P_{i}=1$.

Holm [7] showed that $D \subset \mathcal{D}^{(1)}(\mathcal{A})$, and furthermore that:

Theorem 11. Suppose $P=p_{1} \cdots p_{r}$ is the defining polynomial of a generic arrangement $\mathcal{A}$. Let $M$ and $D$ be as above, and let $I$ be the principal ideal on $P$. Then

$$
\mathcal{D}^{(m)}(\mathcal{A})=\bigoplus_{m \geq 0}\left(\sum_{|\alpha|=m}\left(I:\left(I: P^{\alpha}\right)\right) \delta^{\alpha}+S \varepsilon_{m}\right)
$$

as a bi-graded $S$-module.

Here, the double colon ideal $\left(I:\left(I: P^{\alpha}\right)\right)$ is a principal ideal in $S$, with the generator given by $p_{i_{1}} \cdots p_{i_{\ell}}$, the product of those $p_{j}$ such that some $\delta_{i}$ with $\alpha_{i} \neq 0$ does not annihilate $p_{j}$.

Example 12. Let $p=x y(y-x) \in \mathbb{C}[x, y]=S$. Then the associated arrangement is generic. We get that

$$
P_{1}=y(y-x), \quad P_{2}=x(y-x), \quad P_{3}=x y,
$$

and

$$
\delta_{1}=\partial_{y}, \quad \delta_{2}=\partial_{x}, \quad \delta_{3}=\partial_{x}+\partial_{y} .
$$

Hence $\mathcal{D}^{(0)}(I)=S$, and $\mathcal{D}^{(1)}(I)$ is generated by

$$
y(y-x) \partial_{y}, \quad x(y-x) \partial_{x}, \quad x y\left(\partial_{x}+\partial_{y}\right), \quad x \partial_{x}+y \partial_{y} .
$$

Since any product of distinct $\delta_{i}$ 's is equal to $P=x y(x-y)$, we have that for $m \geq 2$

$\mathcal{D}(I)^{(m)}=S y(y-x) \partial_{y}^{m}+S x(y-x) \partial_{x}^{m}+S x y\left(\partial_{x}+\partial_{y}\right)^{m}+S \varepsilon_{m}+I \mathcal{D}^{(m)}(S)$.

3.3.2. The case $r \leq n$. For the generic arrangement $\mathcal{A}_{n, r}$ with $r \leq n$, we can perform a linear change of variables so that $p_{i}=x_{i}$. Holm 6, Paper III, Proposition 6.2] showed the following:

Proposition 13. $\mathcal{D}^{(m)}\left(x_{1} \cdots x_{r}\right)$ is free with basis $\left\{x_{\alpha} \partial^{\alpha}\left|\alpha \in \mathbb{N}^{n},\right| \alpha \mid=m\right\}$, where $x_{\alpha}$ is the monic generator of $\sqrt{\left\langle x^{\tilde{\alpha}}\right\rangle}$, the radical of $\left\langle x^{\tilde{\alpha}}\right\rangle$, and where

$$
\tilde{\alpha}=\left(\alpha_{1}, \ldots, \alpha_{r}, 0, \ldots, 0\right) \in \mathbb{N}^{n} .
$$

3.3.3. The case $n=2$. Holm [6, Paper III, Prop 6.7] notes that an arrangement $\mathcal{A}$ in $\mathbb{C}^{2}$ is generic. Furthermore, he proves

Proposition 14. Let $\mathcal{A}$ be an arrangement in $\mathbb{C}^{2}$, with defining polynomial $p=p_{1} \cdots p_{r} \in \mathbb{C}\left[x_{1}, x_{2}\right]$, and let $m$ be a positive integer. Put $P_{i}=p / p_{i}$ for $1 \leq i \leq r$, and define

$$
\delta_{i}= \begin{cases}\partial_{2} & \text { if } p_{i}=a x_{1}, a \in \mathbb{C}^{*}, \\ \partial_{1}+a_{i} \partial_{2} & \text { if } p_{i}=a\left(x_{2}-a x_{1}\right), a \in C^{*}\end{cases}
$$

Let

$$
\left\{q_{1}, \ldots, q_{\left(\left(\begin{array}{c}
n \\
m
\end{array}\right)\right)}\right\}=\left\{\partial^{\alpha}|| \alpha \mid=m\right\}
$$


Then $\mathcal{D}^{(m)}(\mathcal{A})$ is free, minimally generated by

$$
\begin{cases}\left\{\varepsilon_{m}, P_{1} \delta_{1}^{m}, \ldots, P_{r} \delta_{r}^{m}\right\} & \text { if } 1 \leq m \leq r-2, \\ \left\{P_{1} \delta_{1}^{r-1}, \ldots, P_{r} \delta_{r}^{r-1}\right\} & \text { if } m=r-1 \\ \left\{P_{1} \delta_{1}^{m}, \ldots, P_{r} \delta_{r}^{m}, p q_{r}, \ldots, p q_{m}\right\} & \text { if } m \geq r .\end{cases}
$$

\section{AN EXACT SEQUENCE}

If $\mathcal{A}$ is an $n$-arrangement consisting of the hyperplanes $H_{1}, \ldots, H_{r}$, recall that the deleted arrangement $\mathcal{A}^{\prime}$ is the arrangement in $\mathbb{C}^{n}$ consisting of the hyperplanes $H_{2}, \ldots, H_{r}$. The restricted arrangement $\mathcal{A}^{\prime \prime}$ is the arrangement $H_{2} \cap H_{1}, \ldots, H_{r} \cap H_{1} \subset H_{1} \simeq \mathbb{C}^{n-1}$. Clearly, if $\mathcal{A}$ is generic then so is $\mathcal{A}^{\prime}$ and $\mathcal{A}^{\prime \prime}$, and we can write

$$
\mathcal{A}_{n, r}^{\prime}=\mathcal{A}_{n, r-1}, \quad \mathcal{A}_{n, r}^{\prime \prime}=\mathcal{A}_{n-1, r-1} .
$$

We can perform a change of coordinates so that the defining polynomial of $H_{1}$ is $x_{n}$. Then multiplication with $x_{n}$ gives a $S$-module homomorphism of degree 1

$$
\mathcal{D}^{(m)}\left(\mathcal{A}_{n, r-1}\right) \stackrel{\cdot x_{n}}{\longrightarrow} \rightarrow \mathcal{D}^{(m)}\left(\mathcal{A}_{n, r}\right),
$$

and the natural projection

$$
\pi: S=\mathbb{C}\left[x_{1}, \ldots, x_{n}\right] \rightarrow S^{\prime}=\frac{S}{x_{n}} \simeq \mathbb{C}\left[x_{1}, \ldots, x_{n-1}\right]
$$

induces a degree 0 map of graded vector spaces

$$
\mathcal{D}^{(m)}\left(\mathcal{A}_{n, r}\right) \rightarrow \mathcal{D}^{(m)}\left(\mathcal{A}_{n-1, r-1}\right)
$$

by replacing every occurrence of $x_{n}$ by zero. The projection $\pi$ can be used to give any $S^{\prime}$ module the structure of $S$-module (via extension of scalars), and so the map (17) becomes an $S$-module homomorphism.

Theorem 15 ([7]). If $m, r, n$ are positive integers with $n>2$, and $\mathcal{A}_{n, r}$ is a generic arrangement, then there is a short exact sequence of graded $S$-modules

$$
0 \rightarrow \mathcal{D}^{(m)}\left(\mathcal{A}_{n, r-1}\right)(-1) \rightarrow \mathcal{D}^{(m)}\left(\mathcal{A}_{n, r}\right) \rightarrow \mathcal{D}^{(m)}\left(\mathcal{A}_{n-1, r-1}\right) \rightarrow 0
$$

Corollary 16. If $m, r, n$ are positive integers with $n>2$ then

$$
\mathcal{H}\left(\mathcal{D}^{(m)}\left(\mathcal{A}_{n, r}\right)\right)(b)=b \mathcal{H}\left(\mathcal{D}^{(m)}\left(\mathcal{A}_{n, r-1}\right)\right)(b)+\mathcal{H}\left(\mathcal{D}^{(m)}\left(\mathcal{A}_{n-1, r-1}\right)\right)(b)
$$

\section{The Hilbert and Poincaré-Betti series of $\mathcal{D}^{(m)}(\mathcal{A})$ FOR GENERIC $\mathcal{A}$}

If $\mathcal{A}$ and $\mathcal{A}^{\prime}$ are two generic $n$-arrangements with $|\mathcal{A}|=\left|\mathcal{A}^{\prime}\right|=r$ then their Hilbert series and Poincaré-Betti series coincide. We let $\mathcal{A}_{n, r}$ denote any generic $n$-arrangement consisting of $r$ hyperplanes. 
5.1. Derivations, the case $m=1$. Yuzvinsky 13 has given a minimal free resolution of $\mathcal{D}^{(1)}\left(\mathcal{A}_{n, r}\right)$.

Theorem 17 (Yuzvinsky). Let $r>n \geq 3$ and let $\mathcal{A}_{n, r}$ be a generic $n$ arrangement with defining polynomial $p=p_{1} \cdots p_{r} \in S=\mathbb{C}\left[x_{1}, \ldots, x_{n}\right]$. Define

$$
\mathcal{D}_{0}=\left\{\theta \in \mathcal{D}^{(1)}\left(\mathcal{A}_{n, r}\right) \mid \theta\left(p_{r}\right)=0\right\} .
$$

Then $\mathcal{D}^{(1)}\left(\mathcal{A}_{n, r}\right)=S \varepsilon_{1} \oplus \mathcal{D}_{0}$ as an $S$-module, and the minimal free resolution of $\mathcal{D}_{0}$ has length $r-1$ and is $r-n+1$-linear. More precisely, the graded Betti numbers of $\mathcal{D}_{0}$ are given by

$$
\beta_{k, u}= \begin{cases}\left(\begin{array}{c}
r \\
n-k
\end{array}\right)\left(\begin{array}{c}
r-n+k-2 \\
k-1
\end{array}\right) & \text { if } u+n-r-1=0 \\
0 & \text { otherwise }\end{cases}
$$

Example 18. If $n=3, r=5$ then the Poincaré-Betti series of $\mathcal{D}_{0}$ is $4 b^{3}+2 b^{4} t$, so the Poincaré-Betti series of $\mathcal{D}^{(1)}\left(\mathcal{A}_{3,5}\right)$ is $b+4 b^{3}+2 b^{4} t$.

We will give a compact formula for the Poincaré-Betti series of derivations, a formula that will give a hint as to what the Poincaré-Betti series of higher order differential operators might look like.

Lemma 19. Let $r>n \geq 3$. Then

$$
\mathcal{P}\left(\mathcal{D}^{(1)}\left(\mathcal{A}_{n, r}\right)\right)(b, t)=b+\left[t^{n-r-1}(1+b t)^{r-1}\right] \odot(1-t)^{n-r}
$$

Example 20. To continue with the previous example, if $n=3, r=5$ then (11) becomes

$$
\begin{aligned}
\mathcal{P}\left(\mathcal{D}^{(1)}\left(\mathcal{A}_{3,5}\right)\right) & =b+\left[t^{-3}(1+b t)^{4}\right] \odot(1-t)^{-2} \\
& =b+\left[t^{-3}+4 b t^{-2}+6 b^{2} t^{-1}+4 b^{3}+b^{4} t\right] \odot\left(1+2 t+3 t^{2}+\ldots\right) \\
& =b+4 b^{3}+2 b^{4} t
\end{aligned}
$$

5.2. The case $n=2$. Proposition 14 shows that when $n=2$, the PoincaréBetti series of $\mathcal{D}^{(m)}\left(\mathcal{A}_{2, r}\right)$ is given by

$$
\mathcal{P}\left(\mathcal{D}^{(m)}\left(\mathcal{A}_{2, r}\right)\right)(b, t)= \begin{cases}r b^{r-1}+(m-r+1) b^{r} & \text { if } r \leq 2 \text { or } m>r-2, \\ b^{m}+m b^{r-1} & \text { otherwise }\end{cases}
$$

It follows that the Hilbert series is

$$
\mathcal{H}\left(\mathcal{D}^{(m)}\left(\mathcal{A}_{2, r}\right)\right)(b, t)= \begin{cases}\frac{r b^{r-1}+(m-r+1) b^{r}}{(1-b)^{2}} & \text { if } r \leq 2 \text { or } m>r-2, \\ \frac{b^{m}+m b^{r-1}}{(1-b)^{2}} & \text { otherwise. }\end{cases}
$$

Together with (91), the initial values (13) determines $\mathcal{H}\left(\mathcal{D}^{(m)}\left(\mathcal{A}_{n, r}\right)\right)(b)$ for all $n \geq 2$.

5.3. The case $r \leq n$. Proposition 13 yields the following: 
Lemma 21. If $r \leq n$ then

$$
\begin{aligned}
\beta_{u, k}^{(m)}\left(\mathcal{A}_{n, r}\right) & = \begin{cases}0 & \text { if } u>0 \\
\left(\begin{array}{l}
r \\
k
\end{array}\right)\left(\left(\begin{array}{c}
n-r+k \\
m-k
\end{array}\right)\right) & \text { if } u=0\end{cases} \\
\mathcal{P}\left(\mathcal{D}\left(\mathcal{A}_{n, r}\right)\right)(a, b, t) & =\frac{(1-a+a b)^{r}}{(1-a)^{n}} \\
\mathcal{H}\left(\mathcal{D}\left(\mathcal{A}_{n, r}\right)\right)(a, b) & =\frac{(1-a+a b)^{r}}{(1-a)^{n}(1-b)^{-n}} \\
\mathcal{P}\left(\mathcal{D}^{(m)}\left(\mathcal{A}_{n, r}\right)(b, t)\right. & =\left[a^{m}\right] \frac{(1-a+a b)^{r}}{(1-a)^{n}} \\
\mathcal{H}\left(\mathcal{D}^{(m)}\left(\mathcal{A}_{n, r}\right)(b)\right. & =\left[a^{m}\right] \frac{(1-a+a b)^{r}}{(1-a)^{n}(1-b)^{-n}}
\end{aligned}
$$

Proof. There are $\left(\begin{array}{l}r \\ k\end{array}\right)$ different square-free $\gamma \in \mathbb{N}^{n}$ of weight $k$ such that

$$
\gamma_{r+1}=\cdots=\gamma_{n}=0
$$

For a fixed such $\gamma$, let $g=g(\gamma)$ denote the number of $\alpha \in \mathbb{N}^{n}$ with $\bar{\alpha}=\gamma$. Then

$$
\alpha \mapsto \alpha-\gamma
$$

gives a bijection between the set of multisets $\alpha$ on $\{1, \ldots, n\}$ with weight $k$ and with $\operatorname{supp}(\tilde{\alpha})=\gamma$, and the set of multisets on

$$
\{r+1, r+2, \ldots, n\} \cup \operatorname{supp}(\gamma)
$$

of weight $m-k$. So $g$ is independent of $\gamma$, and is equal to $\left(\left(\begin{array}{c}n-r+k \\ m-k\end{array}\right)\right)$. It follows that the number of minimal generators of $\mathcal{D}^{m}(\mathcal{A})$ of polynomial degree $k$ is given by $\left(\begin{array}{l}r \\ k\end{array}\right)\left(\left(\begin{array}{c}n-r+k \\ m-k\end{array}\right)\right)$.

To prove the second identity, we take advantage of the fact that we may assume that the linear forms defining the arrangement are the monomials $x_{1}, \ldots, x_{r}$. For this particular arrangement, the modules of differentials will be multi-graded, by giving the operator $x^{\alpha} \partial^{\beta}$ the multi-degree $(\alpha, \beta)$. We will calculate Poincaré-betti series with respect to this fine grading, then specialize to get the desired series (which itself can not be given this fine grading).

We start by calculating the generating function

$$
G\left(a_{1}, \ldots, a_{n}, b_{1}, \ldots, b_{n}\right)
$$

where the sum is over all minimal generators of $\mathcal{D}\left(\mathcal{A}_{n, r}\right)$, and where where $x^{\alpha} \partial^{\beta}$ contributes $a_{1}^{\beta_{1}} \cdots a_{n}^{\beta_{n}} b_{1}^{\alpha_{1}} \cdots b_{n}^{\alpha_{n}}$. Then as before we are looking for all 
pairs $(\alpha, \beta)$ with $\alpha \subset\{0, \ldots, r\}$ and $\operatorname{supp}(\tilde{\alpha})=\beta$. Hence

$$
\begin{aligned}
G\left(a_{1}, \ldots, a_{n}, b_{1}, \ldots, b_{n}\right) & =\sum_{\beta \subseteq\{1,2, \ldots, r\}} b^{\beta}\left(\sum_{\operatorname{supp}(\gamma) \subset \beta} a^{\gamma}\right)\left(\sum_{\operatorname{supp}(\theta) \subseteq\{r+1, \ldots, n\}} a^{\theta}\right) \\
& =\sum_{\beta \subseteq\{1,2, \ldots, r\}} a^{\beta} b^{\beta} \prod_{i=r+1}^{n}\left(1-a_{j}\right)^{-1} \\
& =\prod_{i=1}^{r} \frac{1-a_{i}+a_{i} b_{i}}{1-a_{i}} \prod_{j=r+1}^{n}\left(1-a_{j}\right)^{-1}
\end{aligned}
$$

Specializing gives

$$
P\left(\mathcal{A}_{n, r}\right)(a, b, t)=G(a, \ldots, a, b, \ldots, b)=\frac{(1-a+a b)^{r}}{(1-a)^{n}}
$$

5.4. The cases $m=r-n+1$ and $m>r-n+1$. As we have already noted, it is known that $\mathcal{D}^{(m)}\left(\mathcal{A}_{n, r}\right)$ is free when $r \leq n$ or when $n \leq 2$. Holm [6. Paper III] showed that when $r>n \geq 3, m=r-n+1$, then $\mathcal{D}^{(m)}\left(\mathcal{A}_{n, r}\right)$ is free module, minimally generated by $\left(\begin{array}{c}r \\ n-1\end{array}\right)$ differential operators of order polynomial degree $m$. He conjectured that $\mathcal{D}^{(m)}\left(\mathcal{A}_{n, r}\right)$ is free when $r>n \geq 3$ and $m \geq r-n+1$. More precisely, it is reasonable to conjecture that (14) holds also for this range. This is certainly true for the Hilbert series:

Lemma 22. For $r \leq m+n-1$,

$$
\mathcal{H}\left(\mathcal{D}^{(m)}\left(\mathcal{A}_{n, r}\right)\right)(b)=\left[a^{m}\right] \frac{(1-a+a b)^{r}}{(1-a)^{n}(1-b)^{n}}
$$

Proof. This holds for $n=2$ by (13). Furthermore, if $r \leq m+n-1$ then $r-1 \leq m+n-1$ and $r-1 \leq m+n-1-1$, so the assertion follows by induction, since

$$
\left[a^{m}\right] \frac{(1-a+a b)^{r}}{(1-a)^{n}(1-b)^{n}}=\left[a^{m}\right] \frac{(1-a+a b)^{r-1}}{(1-a)^{n}(1-b)^{n}}+\left[a^{m}\right] \frac{(1-a+a b)^{r-1}}{(1-a)^{n-1}(1-b)^{n-1}} .
$$

5.5. The case $r \geq m+n$. Holm [6] Paper III] showed that when $r>n \geq 3$, $r \geq m+n$, then $\mathcal{D}^{(m)}\left(\mathcal{A}_{n, r}\right)$ is not a free module. This is therefore the "interesting range". We'll eventually formulate a conjecture regarding the Poincaré-Betti series of $\mathcal{D}^{(m)}\left(\mathcal{A}_{n, r}\right)$ for $m, n, r$ in this range.

We'll simplify the problem slightly by identifying a direct summand of these modules. Recall the notations of Theorem [11 so that $D=$ $\left\{P_{1} \delta_{1}, \ldots, P_{t} \delta_{t}\right\}$ is a certain subset of $\mathcal{D}^{(1)}\left(\mathcal{A}_{n, r}\right)$ with the property that

$$
\left\{P_{\alpha} \delta^{\alpha}\left|\alpha \in \mathbb{N}^{t},\right| \alpha \mid=m\right\} \cup\left\{\varepsilon_{m}\right\}
$$

generates $\mathcal{D}^{(m)}\left(\mathcal{A}_{n, r}\right)$, where $P_{\alpha}$ is the product of those $p_{i}$ such that some $\delta_{j}$ with $\alpha_{j} \neq 0$ does not annihilate $p_{i}$.

Let $\Xi^{(m)}\left(\mathcal{A}_{n, r}\right)$ be the module generated by

$$
\left\{P_{\alpha} \delta^{\alpha}\left|\alpha \in \mathbb{N}^{t},\right| \alpha \mid=m\right\} \text {. }
$$


Then Holm's result can be stated as

$$
\mathcal{D}^{(m)}\left(\mathcal{A}_{n, r}\right)=\Xi^{(m)}(\mathcal{A})+S \varepsilon_{m}
$$

Holm showed [6. Paper I, Lemma 5.27] that for $r \leq n, \varepsilon_{m} \in \Xi^{(m)}(\mathcal{A})$. Furthermore, we have:

Lemma 23. Suppose that $r>n \geq 3, r \geq m+n$. Then $\varepsilon_{m} \notin \Xi^{(m)}\left(\mathcal{A}_{n, r}\right)$, so

$$
\mathcal{D}^{(m)}\left(\mathcal{A}_{n, r}\right)=\Xi^{(m)}(\mathcal{A}) \oplus S \varepsilon_{m}
$$

Hence,

$$
\mathcal{P}\left(\mathcal{D}^{(m)}\left(\mathcal{A}_{n, r}\right)\right)(b, t)=b^{m}+\mathcal{P}\left(\Xi^{(m)}\left(\mathcal{A}_{n, r}\right)\right)(b, t)
$$

Proof. All $P_{i}$ have degree $r-n+1$, so all $P_{\alpha}$ have degrees $\geq r-n+1>m$, hence all $P_{\alpha} \delta^{\alpha}$ have polynomial degrees $>m$. But $\varepsilon_{m}$ have polynomial degree $m$, hence can not be expressed as an $S$-linear combination of the $P_{\alpha} \delta^{\alpha}$ 's. This shows that $\varepsilon_{m} \notin \Xi^{(m)}(\mathcal{A})$, which together with (18) yields (19).

Below, we tabulate $\mathcal{P}\left(\Xi^{(m)}\left(\mathcal{A}_{n, r}\right)\right)(b, t)$ for $m=2,3,4$ (these PoincaréBetti series were calculated using Macaulay $2[\underline{5}$ and the method described in the beginning).

For $m=2$, the Poincaré-Betti series are as follows:

\begin{tabular}{|c|ccc|}
\hline $\mathrm{r}$ & $\mathrm{n}=3$ & $\mathrm{n}=4$ & $\mathrm{n}=5$ \\
\hline & & & - \\
5 & $2 b^{4} t+7 b^{3}$ & - & - \\
6 & $4 b^{5} t+9 b^{4}$ & $2 b^{5} t^{2}+9 b^{4} t+16 b^{3}$ & \\
7 & $6 b^{6} t+11 b^{5}$ & $6 b^{6} t^{2}+22 b^{5} t+25 b^{4}$ & $2 b^{6} t^{3}+11 b^{5} t^{2}+25 b^{4} t+30 b^{3}$ \\
8 & $8 b^{7} t+13 b^{6}$ & $12 b^{7} t^{2}+39 b^{6} t+36 b^{5}$ & $8 b^{7} t^{3}+39 b^{6} t^{2}+72 b^{5} t+55 b^{4}$ \\
9 & $10 b^{8} t+15 b^{7}$ & $20 b^{8} t^{2}+60 b^{7} t+49 b^{6}$ & \\
10 & $12 b^{9} t+17 b^{8}$ & & \\
\hline
\end{tabular}

We conjecture that

$\mathcal{P}\left(\Xi^{(2)}\left(\mathcal{A}_{n, r}\right)(b, t)=\left[t^{n-r-1}(1+2 b t)(1+b t)^{r-2}\right] \odot(1-t)^{n-r+1}\right.$

For $m=3$, we get

\begin{tabular}{|c|ccc|}
\hline $\mathrm{r}$ & $\mathrm{n}=3$ & $\mathrm{n}=4$ & $\mathrm{n}=5$ \\
\hline & & & \\
6 & $3 b^{5} t+12 b^{4}$ & - & - \\
7 & $6 b^{6} t+15 b^{5}$ & $3 b^{6} t^{2}+15 b^{5} t+31 b^{4}$ & - \\
8 & $9 b^{7} t+18 b^{6}$ & $9 b^{7} t^{2}+36 b^{6} t+46 b^{5}$ & $3 b^{7} t^{3}+18 b^{6} t^{2}+46 b^{5} t+65 b^{4}$ \\
9 & $12 b^{8} t+21 b^{7}$ & $18 b^{8} t^{2}+63 b^{7} t+64 b^{6}$ & $111 b^{5}+128 t b^{6}+63 t^{2} b^{7}+12 t^{3} b^{8}$ \\
\hline
\end{tabular}

We conjecture that

$$
\begin{aligned}
& \mathcal{P}\left(\Xi^{(3)}\left(\mathcal{A}_{n, r}\right)\right)(b, t)= \\
& {\left[t^{n-r-1}\left(1+3 b t+3 b^{2} t^{2}\right)(1+b t)^{r-3}\right] \odot(1-t)^{n-r+2}}
\end{aligned}
$$

For $m=4$ we get

\begin{tabular}{|c|cc|}
\hline $\mathrm{r}$ & $\mathrm{n}=3$ & $\mathrm{n}=4$ \\
\hline & & \\
7 & $18 b^{5}+4 t b^{6}$ & - \\
8 & $52 b^{5}+22 t b^{6}+4 t^{2} b^{7}$ & $8 b^{7} t+22 b^{6}$ \\
\hline
\end{tabular}


We conjecture that

$$
\begin{aligned}
\mathcal{P}\left(\Xi^{(4)}\left(\mathcal{A}_{n, r}\right)\right)(b, t) & = \\
& {\left[t^{n-r-1}\left(1+4 b t+6 b^{2} t^{2}+4 b^{3} t^{3}\right)(1+b t)^{r-4}\right] \odot(1-t)^{n-r+3} }
\end{aligned}
$$

Based on these computations, we make the following conjecture, which by Yuzvinsky's result is true for derivations, i.e. when $m=1$.

Conjecture 24. Suppose that $3 \leq n$ and $r \geq m+n$. Let $\mathcal{A}_{n, r}$ be a generic $n$-arrangement with $\left|\mathcal{A}_{n, r}\right|=r$. Then

$$
\begin{aligned}
& \mathcal{P}\left(\Xi^{(m)}\left(\mathcal{A}_{n, r}\right)\right)= \\
& \quad\left\{t^{-r+n-1}\left((1+b t)^{m}-(b t)^{m}\right)(1+b t)^{r-m}\right\} \odot(1-t)^{m-r+n-1}
\end{aligned}
$$

and hence

$$
\begin{aligned}
& \mathcal{P}\left(\mathcal{D}^{(m)}\left(\mathcal{A}_{n, r}\right)\right)= \\
& b^{m}+\left\{t^{-r+n-1}\left((1+b t)^{m}-(b t)^{m}\right)(1+b t)^{r-m}\right\} \odot(1-t)^{m-r+n-1}
\end{aligned}
$$

Note that this conjecture implies that

(i) The homological dimension of the $S$-module $\Xi^{(m)}\left(\mathcal{A}_{n, r}\right)$ (and of $\left.\mathcal{D}^{(m)}\left(\mathcal{A}_{n, r}\right)\right)$ is $n-2$.

(ii) The minimal free resolution of $\left.\Xi^{(m)}\left(\mathcal{A}_{n, r}\right)\right)$ is $r-n$-linear.

(iii) $\Xi^{(m)}\left(\mathcal{A}_{n, r}\right)$ is minimally generated by

$$
\left(\begin{array}{c}
r \\
r-n+1
\end{array}\right)-\left(\begin{array}{c}
r-m \\
r-n+1-m
\end{array}\right)=\left(\begin{array}{c}
r \\
n-1
\end{array}\right)-\left(\begin{array}{c}
r-m \\
n-1
\end{array}\right)
$$

differential operators of polynomial degree $r-n+1 ; \mathcal{D}^{(m)}\left(\mathcal{A}_{n, r}\right)$ is minimally generated by these differential operators and $\varepsilon_{m}$.

We now indicate a possible way of proving eq:pdmm. The short exact sequence (8), together with Lemma 23, gives a short exact sequence

$$
0 \rightarrow \Xi^{(m)}\left(\mathcal{A}_{n, r-1}\right)(-1) \rightarrow \Xi^{(m)}\left(\mathcal{A}_{n, r}\right) \rightarrow \Xi^{(m)}\left(\mathcal{A}_{n-1, r-1}\right) \rightarrow 0
$$

which gives rise to a long exact sequence in homology (we have omitted the shifts which are necessary to make the morphisms below homogeneous of degree zero)

$$
\begin{aligned}
\cdots \rightarrow \operatorname{Tor}_{S}^{1}\left(\mathcal{D}^{(m)}\left(\mathcal{A}_{n, r-1}\right), \mathbb{C}\right) \rightarrow \operatorname{Tor}_{S}^{1}\left(\mathcal{D}^{(m)}\left(\mathcal{A}_{n, r}\right), \mathbb{C}\right) \rightarrow \\
\rightarrow \operatorname{Tor}_{S}^{1}\left(\mathcal{D}^{(m)}\left(\mathcal{A}_{n-1, r-1}\right), \mathbb{C}\right) \stackrel{\delta_{1}}{\rightarrow} \Xi^{(m)}\left(\mathcal{A}_{n, r-1}\right) \otimes_{S} \mathbb{C} \rightarrow \\
\rightarrow \Xi^{(m)}\left(\mathcal{A}_{n, r}\right) \otimes_{S} \mathbb{C} \rightarrow \Xi^{(m)}\left(\mathcal{A}_{n-1, r-1}\right) \otimes_{S} \mathbb{C} \rightarrow 0
\end{aligned}
$$

which controls the "deviation"

$q(m, n, r)=\mathcal{P}\left(\Xi^{(m)}\left(\mathcal{A}_{n, r}\right)\right)-b \mathcal{P}\left(\Xi^{(m)}\left(\mathcal{A}_{n, r-1}\right)\right)-(1+b t) \mathcal{P}\left(\Xi^{(m)}\left(\mathcal{A}_{n-1, r-1}\right)\right)$.

If all the connecting homomorphisms $\delta_{i}$ are zero, then so is this deviation, and the Poincaré-Betti series can be computed recursively using deletionrestriction. In the "interesting range" $3 \leq n<r, r \geq m+n$, assuming the conjectured formula (24), and using Lemma 25 below, we do get that 
$q(m, n, r)=0$. This indicates (but does not prove) that the connecting homomorphisms are zero. Conversely, a proof of the vanishing of all connecting homomorphisms would also prove (24).

Lemma 25. For $3 \leq n<r, r \geq m+n$, it holds that

$$
\begin{gathered}
\left\{t^{-r+n-1}\left((1+b t)^{m}-(b t)^{m}\right)(1+b t)^{r-m}\right\} \odot(1-t)^{m-r+n-1} \\
-b\left\{t^{-r+n}\left((1+b t)^{m}-(b t)^{m}\right)(1+b t)^{r-1-m}\right\} \odot(1-t)^{m-r+n} \\
-(1+b t)\left\{t^{-r+n-1}\left((1+b t)^{m}-(b t)^{m}\right)(1+b t)^{r-1-m}\right\} \odot(1-t)^{m-r+n-1}=0
\end{gathered}
$$

Proof of Lemma 25. Put $k=r-n$ and

$$
\begin{aligned}
U(r, k, m) & =\left[\left((1+b t)^{m}-(b t)^{m}\right)(1+b t)^{r-m}\right] \\
& =t^{-k-1}(1+b t)^{r}-t^{m-k-1}(1+b t)^{r-m}
\end{aligned}
$$

Then for $\ell>0$ we have that

$$
\begin{aligned}
{\left[x^{\ell}\right] } & U(r, k, m)=b^{\ell+k+1}\left[\left(\begin{array}{c}
r \\
\ell+k+1
\end{array}\right)-\left(\begin{array}{c}
r-m \\
\ell-m+k+1
\end{array}\right)\right] \\
{\left[x^{\ell}\right] } & b U(r-1, k-1, m)=b^{\ell+k+1}\left[\left(\begin{array}{l}
r-1 \\
\ell+k
\end{array}\right)-\left(\begin{array}{c}
r-1-m \\
\ell-m+k
\end{array}\right)\right] \\
{\left[x^{\ell}\right] } & U(r-1, k, m)=b^{\ell+k+1}\left[\left(\begin{array}{c}
r-1 \\
\ell+k+1
\end{array}\right)-\left(\begin{array}{c}
r-1-m \\
\ell-m+k+1
\end{array}\right)\right] \\
{\left[x^{\ell}\right] } & \text { bt } U(r-1, k, m)=b^{\ell+k+1}\left[\left(\begin{array}{l}
r-1 \\
\ell+k
\end{array}\right)-\left(\begin{array}{c}
r-1-m \\
\ell-m+k
\end{array}\right)\right]
\end{aligned}
$$

Since

$$
(1-t)^{m-r+n-1}=\sum_{\ell=0}^{\infty}\left(\left(\begin{array}{c}
m-r+n-1 \\
\ell
\end{array}\right)\right)
$$

the equation (28) is equivalent to the identity

$$
\begin{aligned}
{\left[\left(\begin{array}{c}
r \\
\ell+k+1
\end{array}\right)-\right.} & \left.\left(\begin{array}{c}
r-m \\
\ell-m+k+1
\end{array}\right)\right]\left(\left(\begin{array}{c}
m-k+1 \\
\ell
\end{array}\right)\right) \\
& -\left[\left(\begin{array}{l}
r-1 \\
\ell+k
\end{array}\right)-\left(\begin{array}{c}
r-1-m \\
\ell-m+k
\end{array}\right)\right]\left(\left(\begin{array}{c}
m-k \\
\ell
\end{array}\right)\right) \\
- & {\left[\left(\begin{array}{c}
r-1 \\
\ell+k+1
\end{array}\right)-\left(\begin{array}{c}
r-1-m \\
\ell-m+k+1
\end{array}\right)\right]\left(\left(\begin{array}{c}
m-k+1 \\
\ell
\end{array}\right)\right) } \\
& -\left[\left(\begin{array}{c}
r-1 \\
\ell+k
\end{array}\right)-\left(\begin{array}{c}
r-1-m \\
\ell-m+k
\end{array}\right)\right]\left(\left(\begin{array}{c}
m-k+1 \\
\ell-1
\end{array}\right)\right)=0,
\end{aligned}
$$

which can be verified algorithmically ${ }^{1}$.

The case $\ell=0$ is dealt with similarly.

\footnotetext{
3 .

${ }^{1}$ We used the simplify (expr, GAMMA) command of the computer algebra system Maple
} 


\section{REFERENCES}

[1] William W. Adams and Philippe Loustaunau. An introduction to Gröbner bases, volume 3 of Graduate Studies in Mathematics. American Mathematical Society, Providence, RI, 1994.

[2] J.-E. Björk. Rings of differential operators, volume 21 of North-Holland Mathematical Library. North-Holland Publishing Co., Amsterdam, 1979.

[3] Bruce W. Char, Keith O. Geddes, Gaston H. Gonnet, Benton L. Leong, Michael B. Monagan, and Stephen M. Watt. Maple V Library reference manual. Springer-Verlag, 1991.

[4] S. C. Coutinho. A primer of algebraic D-modules, volume 33 of London Mathematical Society Student Texts. Cambridge University Press, Cambridge, 1995.

[5] Daniel R. Grayson and Michael E. Stillman. Macaulay 2. Computer algebra program, available at http://www.math.uiuc.edu/Macaulay2/

[6] Pär Holm. Differential Operators on Arrangements of Hyperplanes. PhD thesis, Stockholm University, 2002.

[7] Pär Holm. Differential operators on hyperplane arrangements. Communications in Algebra, 32(6):2177 - 2201, 2004. DOI: 10.1081/AGB-120037213.

[8] Tadeusz Józefiak and Bruce E. Sagan. Basic derivations for subarrangements of Coxeter arrangements. J. Algebraic Combin., 2(3):291-320, 1993.

[9] Joseph P. S. Kung. A geometric condition for a hyperplane arrangement to be free. Adv. Math., 135(2):303-329, 1998.

[10] Peter Orlik and Hiroaki Terao. Arrangements of hyperplanes, volume 300 of Grundlehren der Mathematischen Wissenschaften [Fundamental Principles of Mathematical Sciences]. Springer-Verlag, Berlin, 1992.

[11] Lauren L. Rose and Hiroaki Terao. A free resolution of the module of logarithmic forms of a generic arrangement. Journal of Algebra, 136:376-400, 1991.

[12] Harry Tsai and Anton Leykin. D-modules.m2. Macaulay 2 package, available at http://www2.math.uic.edu/ leykin/Research/Dmodules/Dmods.html 2002.

[13] Sergey Yuzvinsky. A free resolution of the module of derivations for generic arrangements. Journal of Algebra, 136:432-436, 1991.

Department of Mathematics, Stockholm University, SE-10691 Stockholm, SWEDEN

E-mail address: Jan.Snellman@math.su.se 